\title{
9. Sur les Dérivations dans les Espaces Vectoriels Topologiques sur le Corps des Nombres Complexes. III
}

\author{
Par Riichi InNo \\ Université de Waseda
}

(Comm. by K. KUnUGI, M.J.A., Jan. 12, 1960)

Continuons l'étude sur les dérivations dans les espaces vectoriels topologiques sur le corps des nombres complexes. ${ }^{1)}$ Dans cette note, nous désignerons par $E$ et $F$ deux espaces vectoriels topologiques sur le corps $C$ des nombres complexes, localement convexes, séparés et complets. $^{2)}$

1. Théorèmes de l'identité et de Liouville. Commençons par nous préparer un lemme suivant:

Lemme 1.1. Soient $x_{0}$ un point fixé de $E, V, V_{1}$ et $V_{2}$ trois voisinages disqués et ouverts de 0 dans $E$, tels qu'on ait $V \supset V_{1}+V_{1}+V_{1}$ et $V_{1} \supset V_{2}+V_{2}$. Soient $\Omega=x_{0}+V, \Omega_{1}=x_{0}+V_{1}, x_{1}$ un point fixé de $\Omega_{1}$ tel que $x_{1}-x_{0} \in V_{2}, \Omega_{2}=x_{1}+V_{2}$. Si $f$ est une application définie sur $\Omega$ $\grave{a}$ valeurs dans $F$, faiblement dérivable partout dans $\Omega$ au long de $h$ pour tout $h \in E$ et $f=0$ partout dans $\Omega_{2}, f$ doit être aussi égale à 0 identiquement dans $\Omega$.

Démonstration. Remarquons, d'abord, qu'on a $\Omega \supset \Omega_{1} \supset \Omega_{2}$. Soit $x_{2}$ un point arbitraire dans $\Omega_{1}$; comme $V_{1}$ est disqué, on a $x_{2}-x_{1} \in V_{1}+V_{1}$. Puisque $V_{1}+V_{1}$ est aussi un voisinage disqué et ouvert de 0 , il existe un nombre $\alpha(>1)$, tel que, pour tout nombre complexe $t(|t|<\alpha)$, $t\left(x_{2}-x_{1}\right) \in V_{1}+V_{1}$ et par suite $x_{1}+t\left(x_{2}-x_{1}\right)-x_{0} \in V_{1}+V_{1}+V_{1} \subset V$ ont lieu. D'autre part, il existe un nombre $\beta(0<\beta<\alpha)$, tel que la relation $|t|<\beta$ entraîne $t\left(x_{2}-x_{1}\right) \in V_{2}$ ( $V_{2}$ disqué).

Par l'hypothèse, une fonction $\varphi(t)=f\left(x_{1}+t\left(x_{2}-x_{1}\right)\right)$ d'une variable complexe définie dans un domaine $D=\{t ;|t|<\alpha\}$ dans le plan complexe à valeurs dans $F$ est régulière dans $D$ et égale à 0 dans un domaine $D_{1}=\{t ;|t|<\beta\}$ contenu dans $D$. Donc, en vertu du remarque 3.1 (I), pour chaque $y^{\prime} \in F^{\prime}$ (le dual de $F$ ), une fonction numérique $\left\langle\varphi(t), y^{\prime}\right\rangle$ est régulière dans $D$ et égale à 0 dans $D_{1}$, le crochet $\langle$,$\rangle désignant$ la dualité entre $F$ et $F^{\prime}$. Or, dans cette condition, on a $\left\langle\varphi(t), y^{\prime}\right\rangle=0$ partout dans $D$ pour chaque $y^{\prime} \in F^{\prime}$, à cause du théorème de l'identité dans la théorie des fonctions d'une variable complexe. Par conséquent, l'identité $f\left(x_{1}+t\left(x_{2}-x_{1}\right)\right)=0$ a lieu partout dans $D$, en particulier, en

1) R. Iino: Sur les dérivations dans les espaces vectoriels topologiques sur le corps des nombres complexes. I, Proc. Japan Acad., 35, no. 7, 343-348 (1959); Ibid. II, 35, no. 9, 530-535 (1959). Nous noterons par (I) et (II) ces Notes, respectivement.

2) L'hypothèse à la complécité de $E$ n'est pas essentielle, ainsi que dans (I) et (II). 
posant $t=1, f\left(x_{2}\right)=0$, ce qui montre que $f=0$ dans $\Omega_{1}, x$ étant arbitraire dans $\Omega_{1}$.

Enfin, on peut aisément déduire du fait démontré au-dessus que $f=0$ dans $\Omega$.

D'après ce lemme, on a immédiatement le

Théorème 1.1 (Théorème de l'identité). Soient $\Omega$ un domaine dans $E$ (partie ouverte et connexe de $E$ ), $\Omega_{1}$ un sous-domaine non vide de $\Omega, f$ une application de $\Omega$ dans $F$, faiblement dérivable partout dans $\Omega$ au long de $h$ pour tout élément $h$ de $E$. Dans ces conditions, lorsque $f=0$ dans $\Omega_{1}$, on a $f=0$ dans $\Omega$.

Démonstration. Soit $A$ un ensemble de tout point de $\Omega$, tel qu'il existe un voisinage ouvert de ce point dans lequel $f=0 ; A$ est évidemment un ensemble ouvert dans $\Omega$. Nous allons maintenant démontrer qu'il est à la fois un ensemble fermé dans $\Omega$. Soit $x_{0}$ un point adhérent à $A$; quel que soit $V$ un voisinage disqué et ouvert de 0 , tel que $x_{0}+V \subset \Omega$, il existe un voisinage disqué et ouvert $V_{1}$ de 0 , tel que $V_{1}+V_{1}+V_{1} \subset V$, et il existe un point $x_{1}$ qui appartient à $A \frown\left(x_{0}+V_{1}\right)$. D'après la définition de $A$, il existe un voisinage disqué et ouvert $V_{2}$ de 0 , tel qu'on ait $x_{1}+V_{2} \subset A \cap\left(x_{0}+V_{1}\right)$ et donc $f=0$ ait lieu dans $x_{1}+V_{2}$. Par conséquent, d'après le lemme précédent, il en résulte que $f=0$ dans $x_{0}+V$, donc $x_{0} \in A$, qui montre bien que $A$ est fermé dans $\Omega$. Comme $\Omega$ est connexe et $A$ est non vide, on a finalement $\Omega=A$, ce qui achève la démonstration.

On va maintenant étendre le théorème de Liouville:

Théorème 1.2 (Théorème de Liouville). Soit $f$ une application de $E$ dans $F$, faiblement dérivable partout dans $E$ au long de $h$ pour tout $h$ dans $E$. Si $f$ est borné dans $E$, on a $f(x)=$ constante $\in F$ pour tout $x$ de $E$.

En effet, soient $x$ un point arbitraire de $E, t$ une variable complexe; la fonction $\varphi(t)=f(t x)$ est une fonction entière bornée d'une variable complexe à valeurs dans $F$. En vertu du théorème généralisé de Liouville, ${ }^{3)}$ on a immédiatement $\varphi(t)=$ constante, en particulier, $f(x)=$ $\varphi(1)=\varphi(0)=f(0)$, d'où le théorème, $x$ étant arbitraire dans $E$.

2. Structure uniforme sur l'espace $D(\Omega, F)$. Soit $\Omega$ une partie ouverte de $E$. Nous désignerons par $D(\Omega, F)$ l'ensemble de toutes les applications continues de $\Omega$ dans $F$, faiblement dérivables partout dans $\Omega$ au long de $h$ pour tout $h \in E$. D'après les théorèmes 3.1 (I) et 3.2 (II), on peut dire que $D(\Omega, F)$ est l'ensemble de toutes les applications de $\Omega$ dans $F$, dérivables au sens de Fréchet partout dans $\Omega$. Nous avons su le fait que, dans le théorème 2.1 (I), l'ensemble $D(\Omega, F)$ est un espace vectoriel sur $C$.

Nous allons maintenant introduire dans l'ensemble $D(\Omega, F)$ la $\subseteq$ -

3) T. Shibata: Sci. Rep. Tokyo Kyoiku Daigaku, 5, no. 133 (1955). 
topologie.) Étant donné un ensemble $\subseteq$ des parties non vides de $E$, tel que tout élément de $\Omega$ appartienne à un ensemble de $\subseteq$ au moins, nous désignerons par $D_{\Im}(\Omega, F)$ l'espace topologique obtenu en munissant $D(\Omega, F)$ de la S-topologie; la S-topologie sur $D(\Omega, F)$ est compatible avec la structure de groupe additif de $D(\Omega, F)$. Puisque l'espace $F$ est un espace uniforme et séparé, il s'ensuit que l'espace topologique $D_{\Phi}(\Omega, F)$ est aussi uniforme et séparé.

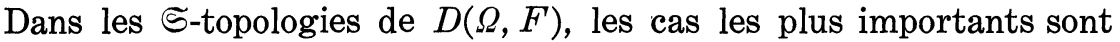
les suivants:

1) $\subseteq$ est l'ensemble de toutes les parties compactes de $\Omega$ (la topologie de la convergence compacte). ${ }^{5}$

2) $\subseteq$ est l'ensemble formé de la seule partie $\Omega$ (la topologie de la convergence uniforme).

Il est facile de voir que la structure uniforme 1 ) de $D(\Omega, F)$ (se note $D_{c}(\Omega, F)$ ) est compatible avec la structure d'espace vectoriel sur $C$ de $D(\Omega, F){ }^{6}{ }^{6}$ Au contraire, en général, la structure uniforme 2) de $D(\Omega, F)$ n'est pas compatible avec la structure uniforme d'espace vectoriel sur $C$ de $D(\Omega, F)$.

Considérons d'abord l'espace $D_{u}(\Omega, F)$ obtenu en munissant $D(\Omega, F)$ de la topologie de la convergence uniforme sur $\Omega$. On va montrer le

Théorème 2.1. L'espace $D_{u}(\Omega, F)$ est complet.

Démonstration. Désignerons par $\mathscr{F}_{u}(\Omega, F)$ l'espace uniforme de toutes les applications de $\Omega$ dans $F$, muni de la topologie de la convergence uniforme sur $\Omega$. On sait que $\mathscr{F}_{u}(\Omega, F)$ est un espace complet; ${ }^{7)}$ tout revient à démontrer que l'espace $D_{u}(\Omega, F)$ est un sous-espace fermé de $\mathscr{F}_{u}(\Omega, F)$. Soit $f$ un point adhérent à $D(\Omega, F)$ ( $f$ est nécessairement continue dans $\Omega$ ); quel que soit $V$ un voisinage disqué et fermé de 0 dans $F$, il existe un élément $g \in D(\Omega, F)$, tel qu'on ait

$$
f(x)-g(x) \in V
$$

pour tout $x \in \Omega$.

Soient $x_{0}$ un point fixé de $\Omega$ et $h$ de $E$; il existe un nombre $\alpha>0$, tel que la relation $|t|<\alpha$ ( $t$ un nombre complexe) entraîne $x_{0}+t h \in \Omega$. Donc on a $f\left(x_{0}+t h\right)-g\left(x_{0}+t h\right) \in V$ pour tout $t(|t|<\alpha)$. Par conséquent, lorsque $t_{0}$ est un nombre complexe, tel que $\left|t_{0}\right|<\alpha, r$ un nombre positif, tel que $\alpha-\left|t_{0}\right|>r$, et $t$ un nombre complexe tel que $\left|t-t_{0}\right|<\frac{r}{2}$, la relation suivante a lieu:

$$
\frac{f\left(x_{0}+\xi h\right)}{\xi-t}-\frac{g\left(x_{0}+\xi h\right)}{\xi-t} \in \frac{2}{r} V, \quad \text { pour tout } \xi \text { avec }\left|\xi-t_{0}\right|=r,
$$

puisque $V$ est un voisinage disqué de 0 .

4) N. Bourbaki: Topologie Générale, Chap. X, Paris (1949).

5) On peut remplacer $\subseteq$ par l'ensemble de toutes les parties relativement compactes de $\Omega$.

6) N. Bourbaki: Espaces Vectoriels Topologiques, Chap. III, Paris (1955).

7) Voir N. Bourbaki: Loc. cit. 4), pp. 7-8. 
D'autre part, comme une application $x \rightarrow f(x)$ de $\Omega$ dans $F$ est continue, la fonction $\xi \rightarrow f\left(x_{0}+\xi h\right)$ est aussi une fonction continue $d u$ cercle $\left\{\xi ;\left|\xi-t_{0}\right|=r\right\}$ dans $F$. On a alors, à cause de la relation (2),

$$
\frac{1}{2 \pi i} \int_{\left|\xi-t_{0}\right|=r} \frac{f\left(x_{0}+\xi h\right)}{\xi-t} d \xi-\frac{1}{2 \pi i} \int_{\left|\xi-t_{0}\right|=r} \frac{g\left(x_{0}+\xi h\right)}{\xi-t} d \xi \in 2 V,
$$

car $V$ est fermé et disqué.

Il résulte finalement de (1), (3) et $g \in D(\Omega, F)$ que

$$
\frac{1}{2 \pi i} \int_{\left|\xi-t_{0}\right|=r} \frac{f\left(x_{0}+\xi h\right)}{\xi-t} d \xi-f\left(x_{0}+t h\right) \in 3 V .
$$

Or, comme l'espace $F$ est séparé, on a alors

(5) $f\left(x_{0}+t h\right)=\frac{1}{2 \pi i} \int_{\left|\xi-t_{0}\right|=r} \frac{f\left(x_{0}+\xi h\right)}{\xi-t} d \xi$ pour tout $t$ avec $\left|t-t_{0}\right|<\frac{r}{2}$.

Comme $t_{0}$ a été arbitraire dans un domaine $D=\{t ;|t|<\alpha\}$, la relation (5) signifie que la fonction $f\left(x_{0}+t h\right)$ est régulière dans $D$ et par suite $f \in D(\Omega, F)$ ( $f$ étant continue dans $\Omega$ ), puisque $(x, h)$ est arbitraire dans $\Omega \times E$, c.q.f.d.

Considérons ensuite l'espace $D_{c}(\Omega, F)$ obtenu en munissant $D(\Omega, F)$ de la topologie de la convergence compacte. On a tout de suite le

Théorème 2.2. L'espace $D_{c}(\Omega, F)$ est un espace vectoriel topologique sur le corps $C$, localement convexe et séparé.

En effet, comme nous avons déjà vu, la structure uniforme de l'espace $D_{c}(\Omega, F)$ est compatible avec la structure d'espace vectoriel sur $C$; il suffit donc de montrer que l'espace $D_{c}(\Omega, F)$ est localement convexe, étant ce séparé. Or, cette assertion résulte immédiatement $\mathrm{du}$ fait que l'espace $F$ est localement convexe, c.q.f.d.

Désignerons par $\mathscr{H}_{c}(\Omega, F)$ l'espace uniforme de toutes les applications de $\Omega$ dans $F$, muni de la topologie de la convergence compacte. Tout d'abord, remarquons que l'espace $\mathscr{F}_{c}(\Omega, F)$ est un espace complet et la topologie induite sur $D(\Omega, F)$ par celle de $\mathscr{F}_{c}(\Omega, F)$ est identique à la topologie de $D_{c}(\Omega, F)$. Ensuite, nous désignerons par $K(\Omega, F)$ l'ensemble de toutes les applications de $\Omega$ dans $F$ dont la restriction à toute partie compacte $A$ de $\Omega$ est continue dans $A$ et faiblement dérivable partout dans $\Omega$ au long de $h$ pour tout $h \in E$.

Nous allons démontrer le

Théorème 2.3. L'espace $K_{c}(\Omega, F)$, muni de la topologie induite par celle de $\mathscr{F}_{c}(\Omega, F)$ est un espace vectoriel topologique sur $C$, localement convexe, séparé et complet.

Démonstration. Il suffit de montrer que l'espace $K_{c}(\Omega, F)$ est complet, puisque la partie restante de l'énoncé est évidente. Pour cela, nous allons voir que $K_{c}(\Omega, F)$ est fermé dans $\mathscr{F}_{c}(\Omega, F)$ (l'espace complet). Soit $f$ un point adhérent à $K(\Omega, F) ; f$ est une application de $\Omega$ dans $F$, dont la restriction à toute partie compacte $A$ de $\Omega$ est continue 
dans $A$. En effet, soient $V$ un voisinage de 0 dans $F, W$ un voisinage symétrique de 0 dans $F$ tel que $W+W+W \subset V$; pour toute partie compacte $A$ de $\Omega$, il existe un élément $g \in K(\Omega, F)$, tel qu'on a $f(x)$ $-g(x) \in W$ pour tout $x \in A$, et un voisinage $U(x)$ de $x \in A$, tel que la relation $x^{\prime} \in A \frown U(x)$ entraîne $g(x)-g\left(x^{\prime}\right) \in W$. Par conséquent, on a $f(x)-f\left(x^{\prime}\right) \in W+W+W \subset V$ pour tout $x^{\prime} \in A \frown U(x)$, ce qui montre le fait que la restriction de $f$ à $A$ est continue dans $A$.

D'autre part, $f$ est faiblement dérivable partout dans $\Omega$. En effet, soient $(x, h)$ un point quelconque de $\Omega \times E, V$ un voisinage disqué et fermé de 0 dans $F$. Désignerons par $\alpha$ un nombre positif tel que $\left\{x_{0}+t h ;|t|<\alpha\right\} \subset \Omega$. Donc, pour un nombre positif $r(<\alpha)$, l'ensemble $\left\{x_{0}+t h ;|t| \leq r\right\}$ est une partie compacte de $\Omega$. Par l'hypothèse, il existe un élément $g \in K(\Omega, F)$, tel qu'on ait $f\left(x_{0}+t h\right)-g\left(x_{0}+t h\right) \in V$ pour tout $t(|t| \leq r)$; comme $f\left(x_{0}+t h\right)$ est continue dans l'ensemble $\{t ;|t|<r\}$ et $g\left(x_{0}+t h\right)$ est régulière dans un domaine $\{t ;|t|<\alpha\}$, de la même considération que le théorème 2.1 nous pouvons montrer que la fonction $f\left(x_{0}+t h\right)$ est dérivable en $t=0$, ce qui montre que $f$ est faiblement dérivable en $x_{0}$ au long de $h$, c.q.f.d.

Remarque. Puisque l'espace $D_{c}(\Omega, F)$ est un sous-espace de $K_{c}(\Omega, F)$, $K_{c}(\Omega, F)$ contient le complété $\widehat{D}_{c}(\Omega, F)$ de $D_{c}(\Omega, F)$.

Lorsque l'espace $E$ est localement compact, on peut aisément montrer que les trois espaces $K_{c}(\Omega, F), D_{c}(\Omega, F)$ et $\widehat{D}_{c}(\Omega, F)$ sont identiques. Or, en vertu du théorème bien connu dans la théorie des espaces vectoriels topologiques, un espace vectoriel topologique localement compact sur $C$ doit être de dimension finie sur $C$. Donc, comme dans ce cas $E$ est isomorphe à $C^{n}$ (l'espace numérique de dimension complexe $n$ ), l'identité des trois espaces au-dessus est le fait évident, puisque dans $C^{n}$ la notion de la dérivée au sens de Fréchet (ou de la dérivée faible) à la fonction continue se ramène à la notion de la dérivée usuelle dans la théorie des fonctions à valeurs vectorielles de plusieurs variables complexes.

Lorsque l'espace $E$ est un espace métrisable, c'est-à-dire, un espace de Fréchet, chaque élément $f$ de $K(\Omega, F)$ est nécessairement une fonction continue de $\Omega$ dans $F$. On a donc $K(\Omega, F) \subset D(\Omega, F)$. Par conséquent, on a $K_{c}(\Omega, F)=\widehat{D}_{c}(\Omega, F)=D_{c}(\Omega, F)$, ce qui montre que l'espace $D_{c}(\Omega, F)$ est un espace complet.

3. Continuité de l'application $f \rightarrow f^{\prime}$. Désignerons par $\mathcal{L}(E, F)$ l'espace vectoriel topologique sur $C$ des applications linéaires continues de $E$ dans $F$. D'après le théorème 2.1 (I), l'application $f \rightarrow f^{\prime 8}$ de $D(\Omega, F)$ dans $\mathcal{F}(\Omega, \mathcal{L}(E, F)$ ) (l'espace vectoriel complexe de toutes les applications de $\Omega$ dans $\mathcal{L}(E, F)$ ) est linéaire. D'autre part, en vertu du théorème 3.1 (II), l'application $x \rightarrow f^{\prime}(x)$ de $\Omega$ dans $\mathcal{L}(E, F)$ est une

8) Voir $n^{\circ} 2(I)$. 
application continue de $\Omega$ dans $\mathcal{L}_{b}(E, F)$ (l'espace vectoriel topologique obtenu en munissant $\mathcal{L}(E, F)$ de la topologie de la convergence bornée), c'est-à-dire, $f^{\prime} \in \mathcal{C}\left(\Omega, \mathcal{L}_{b}(E, F)\right.$ ) (l'espace vectoriel sur $C$ des applications continues de $\Omega$ dans $\mathcal{L}_{b}(E, F)$ ).

Nous allons démontrer maintenant le

Théorème 3.1. L'application $f \rightarrow f^{\prime}$ de $D_{u}(\Omega, F)$ dans un espace vectoriel topologique complexe $\mathcal{C}\left(\Omega, \mathcal{L}_{b}(E, F)\right)$, muni de la topologie de la convergence compacte (se note $\mathcal{C}_{c}\left(\Omega, \mathcal{L}_{b}(E, F)\right)$ ), est continue.

Démonstration. Soit $A$ une partie compacte de $E$ contenue dans $\Omega$; pour chacun de point $x \in A$, il existe deux voisinages disqués $W(x)$ et $V(x)$ de 0 dans $E$ tels que $x+W(x) \subset \Omega, V(x)+V(x) \subset W(x)$ et $W(x)$ fermé. Par conséquent, les ensembles $x+V(x)$ forment un recouvrement de $A$, lorsque $x$ parcourt dans $A$; il existe un nombre fini de points $x_{i}(1 \leq i \leq n)$ de $A$ et de voisinages $V_{i}^{9)}(1 \leq i \leq n)$ de 0 tels que $\Omega \supset \bigcup_{i=1}^{n}\left(x_{i}+V_{i}\right) \supset A, A$ étant un ensemble compact.

D'autre part, soit $B$ une partie bornée de $E$; il existe $n$ nombres $\alpha_{i}>0(1 \leq i \leq n)$, dont les relations $|t| \leq \alpha_{i}$ entraînent $t B \subset V_{i}(1 \leq i \leq n)$. Par suite on a $x+t B \subset x_{i}+V_{i}+V_{i} \subset x_{i}+W_{i}(1 \leq i \leq n)$ pour tout $t$ $\left(|t| \leq \alpha_{i}\right)$, lorsque $x$ parcourt dans $x_{i}+V_{i}(1 \leq i \leq n)$, et en posant $\alpha=\operatorname{Min}_{1 \leq i \leq n} \alpha_{i}$, on a $x+t B \subset \bigcup_{i=1}^{n}\left(x_{i}+W_{i}\right) \subset \Omega$, pour tout $x \in A$. Soient maintenant $f$ un élément de $D(\Omega, F), V$ un voisinage disqué et fermé de 0 dans $F$. En posant:

$$
W=\{g \in D(\Omega, F) ; g(x)-f(x) \in \alpha V \quad \text { pour tout } x \in \Omega\}
$$

(un voisinage de $f$ dans $D_{u}(\Omega, F)$ ), pour tout $g \in W$, on a:

$g(x+t h)-f(x+t h) \in \alpha V$, pour tout $x+t h \in A+t B$ avec $|t| \leq \alpha:$

pour tout $\xi(|\xi|=\alpha)$, on a

$$
\frac{g(x+\xi h)}{\xi^{2}}-\frac{f(x+\xi h)}{\xi^{2}} \in \frac{1}{\alpha} V \quad \text { pour tous } x \in A \text { et } h \in B
$$

( $V$ étant disqué), et par suite on a

$$
\frac{1}{2 \pi i} \int_{|\xi|=\alpha} \frac{g(x+\xi h)}{\xi^{2}} d \xi-\frac{1}{2 \pi i} \int_{|\xi|=\alpha} \frac{f(x+\xi h)}{\xi^{2}} d \xi \in V,
$$

$V$ étant fermé. Par conséquent, en vertu de la formule (3) (II), $g \in W$ entraîne $D g(x)[h]-D f(x)[h] \in V$ pour tous $x \in A$ et $h \in B$, ce qui achève la démonstration, compte tenu du théorème 3.2 (II).

Remarque. En général, l'application $f \rightarrow f^{\prime}$ de $D_{u}(\Omega, F)$ dans $\mathcal{C}_{u}\left(\Omega, \mathcal{L}_{b}(E, F)\right)$ n'est pas continue.

De même considération que le théorème au-dessus et quelque peu de la modification, on a le

Théorème 3.2. L'application $f \rightarrow f^{\prime}$ de l'espace vectoriel topologique $D_{c}(\Omega, F)$ dans $\mathcal{C}_{c}\left(\Omega, \mathcal{L}_{c}(E, F)\right)^{10)}$ est une application linéaire continue.

9) On désigne par $V_{i}$ le voisinage $V\left(x_{i}\right)$ de 0.

10) $\operatorname{Par} \mathcal{L}_{c}(E, F)$ on désigne l'espace vectoriel topologique obtenu en munissant $\mathcal{L}(E, F)$ de la topologie de la convergence compacte. 